(C) 1998 Technika

\title{
PARTIAL LINEARIZATION AND PARTIAL DECOUPLING OF NON-INVERTABLE IMPULSIVE EXTENSIONS
}

\author{
LELDE SERMONE \\ Institute of Mathematics and University of Latvia \\ Akademijas laukums 1, Rīga, LV-1524 LATVIA \\ E-mail: lelde@tech.swh.lv
}

\begin{abstract}
A problem of a partial linearization and partial decoupling of non-invertable impulsive extensions is considered. The sufficient conditions for the dynamical equivalence are given.
\end{abstract}

\section{INTRODUCTION}

The theory of differential equations with impulse effect has been developing rapidly in the recent years. These equations are convenient mathematical models for description of evolutionary processes which suddenly change their state at certain moments. The general theory of impulsive systems is described in the following monographs written by D. D. Bainov and S. I. Kostadinov [1], A. M. Samoilenko and N. A. Perestjuk [4]. The classification problems of impulse systems have been considered by A. Reinfelds [2], [3]. This article is worked out on the basis of the previous publications [5], [6].

\section{PROBLEM STATEMENT}

Let $\mathbf{X}$ and $\mathbf{Y}$ be Banach spaces. Consider the following system of differential equations with impulse effect at fixed moments:

$$
\left\{\begin{aligned}
d x / d t & =A(\theta(t, \mu)) x+f(x, y, \theta(t, \mu)), \\
d y / d t & =B(\theta(t, \mu)) y+g(x, y, \theta(t, \mu)), \\
\left.\Delta x\right|_{t=\tau_{i}} & =C_{i}(\mu) x\left(\tau_{i}-0\right)+I_{i}\left(x\left(\tau_{i}-0\right), y\left(\tau_{i}-0\right), \mu\right), \\
\left.\Delta y\right|_{t=\tau_{i}} & =D_{i}(\mu) y\left(\tau_{i}-0\right)+K_{i}\left(x\left(\tau_{i}-0\right), y\left(\tau_{i}-0\right), \mu\right),
\end{aligned}\right.
$$

where: 
(i) $\theta: \mathbb{R} \times \mathbf{M} \rightarrow \mathbf{M}$ is a dynamical system and $\mathbf{M}$ is a topological space;

(ii) the maps $A: \mathbf{M} \rightarrow \mathcal{L}(\mathbf{X}), B: \mathbf{M} \rightarrow \mathcal{L}(\mathbf{Y})$ are locally integrable in Bochner's sense, where $\mathcal{L}(\mathbf{X})$ and $\mathcal{L}(\mathbf{Y})$ are Banach spaces of linear bounded maps;

(iii) the maps $f: \mathbf{X} \times \mathbf{Y} \times \mathbf{M} \rightarrow \mathbf{X}, g: \mathbf{X} \times \mathbf{Y} \times \mathbf{M} \rightarrow \mathbf{Y}$ are locally integrable in Bochner's sense with respect to $\mu$ for fixed $x$ and $y$ and they satisfy the Lipschitz conditions with small $\varepsilon$ uniformly with respect to $\mu$ :

$$
\begin{aligned}
& \left|f(x, y, \mu)-f\left(x^{\prime}, y^{\prime}, \mu\right)\right| \leq \varepsilon\left(\left|x-x^{\prime}\right|+\left|y-y^{\prime}\right|\right), \\
& \left|g(x, y, \mu)-g\left(x^{\prime}, y^{\prime}, \mu\right)\right| \leq \varepsilon\left(\left|x-x^{\prime}\right|+\left|y-y^{\prime}\right|\right),
\end{aligned}
$$

and, in addition,

$$
\sup _{x, y, \mu}|f(x, y, \mu)|<+\infty ;
$$

(iv) $i \in \mathbf{N}, C_{i} \in \mathcal{L}(\mathbf{X}), D_{i} \in \mathcal{L}(\mathbf{Y})$, the maps $I_{i}: \mathbf{X} \times \mathbf{Y} \times \mathbf{M} \rightarrow \mathbf{X}$ and $K_{i}: \mathbf{X} \times \mathbf{Y} \times \mathbf{M} \rightarrow \mathbf{Y}$ satisfy the Lipschitz conditions

$$
\begin{gathered}
\left|I_{i}(x, y, \mu)-I_{i}\left(x^{\prime}, y^{\prime}, \mu\right)\right| \leq \varepsilon\left(\left|x-x^{\prime}\right|+\left|y-y^{\prime}\right|\right), \\
\left|K_{i}(x, y, \mu)-K_{i}\left(x^{\prime}, y^{\prime}, \mu\right)\right| \leq \varepsilon\left(\left|x-x^{\prime}\right|+\left|y-y^{\prime}\right|\right),
\end{gathered}
$$

and, in addition,

$$
\sup _{i, x, y, \mu}\left|I_{i}(x, y, \mu)\right|<+\infty ;
$$

(v) the map $x \mapsto x+C_{i}(\mu) x$ is homeomorphism;

(vi) the moments $\tau_{i}$ of impulse effect form a strictly increasing sequence and

$$
\lim _{n \rightarrow \infty} \tau_{n}=+\infty
$$

Let $\Phi\left(\cdot, t_{0}, x_{0}, y_{0}, \mu\right):\left[t_{0},+\infty\right) \rightarrow \mathbf{X} \times \mathbf{Y}$ be the solution of the system (2.1), which satisfies the initial condition $\Phi\left(t_{0}+0, t_{0}, x_{0}, y_{0}, \mu\right)=\left(x_{0}, y_{0}\right)$ and $\Phi\left(t, t_{0}, x_{0}, y_{0}, \mu\right)=\left(x\left(t, t_{0}, x_{0}, y_{0}, \mu\right), y\left(t, t_{0}, x_{0}, y_{0}, \mu\right)\right)$. At the break points $\tau_{i}$ the values of all solutions are taken at $\tau_{i}-0$, if it is not specified otherwise.

Let $U(t, \tau, \mu)$ and $V(t, \tau, \mu)$ be the Cauchy evolutionary maps of the corresponding linear impulsive systems:

$$
\begin{gathered}
\left\{\begin{aligned}
d U / d t & =A(\theta(t, \mu)) U, \\
\left.\Delta U\right|_{t=\tau_{i}} & =C_{i}(\mu) U\left(\tau_{i}-0\right),
\end{aligned}\right. \\
\left\{\begin{aligned}
d V / d t & =B(\theta(t, \mu)) V, \\
\left.\Delta V\right|_{t=\tau_{i}} & =D_{i}(\mu) V\left(\tau_{i}-0\right) .
\end{aligned}\right.
\end{gathered}
$$


In addition we assume that they satisfy the following equalities:

$$
\begin{gathered}
L=\sup _{t_{0}, \mu}\left(\int_{t_{0}}^{+\infty}\left|U\left(t_{0}, \tau, \mu\right)\right|\left|V\left(\tau, t_{0}, \mu\right)\right| d \tau\right. \\
\left.+\sum_{t_{0} \leq \tau_{i}}\left|U\left(t_{0}, \tau_{i}, \mu\right)\right|\left|V\left(\tau_{i}-0, t_{0}, \mu\right)\right|\right)<\infty \\
M=\sup _{t_{0}, \mu}\left(\int_{t_{0}}^{+\infty}\left|U\left(t_{0}, \tau, \mu\right)\right| d \tau+\sum_{t_{0} \leq \tau_{i}}\left|U\left(t_{0}, \tau_{i}, \mu\right)\right|\right)<\infty .
\end{gathered}
$$

Let us consider (2.1) and the system:

$$
\left\{\begin{aligned}
d x / d t & =A(\theta(t, \mu)) x \\
d y / d t & =B(\theta(t, \mu)) y+g(x+v(t, x, y, \theta(t, \mu)), y, \theta(t, \mu)) \\
\left.\Delta x\right|_{t=\tau_{i}} & =C_{i} x\left(\tau_{i}-0\right) \\
\left.\Delta y\right|_{t=\tau_{i}} & =D_{i} y\left(\tau_{i}-0\right)+K_{i}\left(x\left(\tau_{i}-0\right)\right. \\
& \left.+v\left(\tau_{i}-0, x\left(\tau_{i}-0\right), y\left(\tau_{i}-0\right), \theta\left(\tau_{i}, \mu\right)\right), y\left(\tau_{i}-0\right), \mu\right) .
\end{aligned}\right.
$$

DEFinition 2.1. Two systems of impulsive differential equations (2.1) and (2.4) are dynamically equivalent if there exists the map $H: \mathbb{R} \times \mathbf{X} \times \mathbf{Y} \times \mathbf{M} \rightarrow$ $\mathbf{X} \times \mathbf{Y}$ with the following properties:

(i) $H(t, \cdot, \cdot, \mu): \mathbb{R} \times \mathbf{X} \times \mathbf{Y} \times \mathbf{M} \rightarrow \mathbf{X} \times \mathbf{Y}$ is homeomorphism;

(ii) $H\left(t, \Phi\left(t, t_{0}, x_{0}, y_{0}, \mu\right), \theta\left(t-t_{0}, \mu\right)\right)=\Psi\left(t, t_{0}, H\left(t_{0}, x_{0}, y_{0}, \mu\right), \mu\right)$, if $t \geq$ $t_{0}$, where $\Psi:\left[t_{0},+\infty\right) \rightarrow \mathbf{X} \times \mathbf{Y}$,

$\Psi\left(t, t_{0}, x_{0}, y_{0}, \mu\right)=\left(x_{0}\left(t, t_{0}, x_{0}, y_{0}, \mu\right), y_{0}\left(t, t_{0}, x_{0}, y_{0}, \mu\right)\right)$ is the solution of the system (2.4);

(iii) if the system (2.1) is autonomous and without impulses, then $H$ does not depend on $t$.

\section{THE MAIN THEOREM}

THEOREM 3.1. Let the hypotheses (i)-(vi) be fulfilled, and suppose the inequalities $4 L \varepsilon \leq 1$ and $2 M \varepsilon<1+\sqrt{1-4 L \varepsilon}$ be satisfied, where the constants $L$ and $M$ are specified by formulas (2.2) and ((2.3). Then (2.1) and (2.4) are dynamically equivalent.

Proof. Step 1. Let us consider the Banach space $\mathbf{B}_{1}$ of the bounded maps that are continuous for $(t, x, y, \mu) \in\left(\tau_{i}, \tau_{i+1}\right] \times \mathbf{X} \times \mathbf{Y} \times \mathbf{M}$ and have first kind breaks for $t=\tau_{i}$ :

$$
\mathbf{B}_{1}=\left\{v \mid v: \mathbb{R} \times \mathbf{X} \times \mathbf{Y} \times \mathbf{M} \rightarrow \mathbf{X} \text { and } \sup _{t, x, y, \mu}|v(t, x, y, \mu)|<\infty\right\}
$$


with the norm $\|v\|=\sup _{t, x, y, \mu}|v(t, x, y, \mu)|$.

Let us introduce the set:

$$
\mathbf{G}_{1}(p)=\left\{v \in \mathbf{B}_{1}|| v(t, x, z, \mu)-v\left(t, x, z^{\prime}, \mu\right)|\leq p| z-z^{\prime} \mid\right\}
$$

$\mathbf{G}_{1}(p)$ is a closed subset of the Banach space $\mathbf{B}_{1}$. In $\mathbf{G}_{1}(p)$ we consider the functional equation:

$$
\begin{gathered}
v_{1}\left(t_{0}, x_{0}, z, \theta\left(t_{0}, \mu\right)\right)=-\int_{t_{0}}^{+\infty} U\left(t_{0}, \tau, \mu\right) f\left(x_{0}(\tau)\right. \\
\left.+v_{1}\left(\tau, x_{0}(\tau), z(\tau), \theta(\tau, \mu)\right), z(\tau), \theta(\tau, \mu)\right) d \tau \\
-\sum_{t_{0} \leq \tau_{i}} U\left(t_{0}, \tau_{i}, \mu\right) I_{i}\left(x_{0}\left(\tau_{i}-0\right)\right. \\
\left.+v_{1}\left(\tau_{i}-0, x_{0}\left(\tau_{i}-0\right), z\left(\tau_{i}-0\right), \theta\left(\tau_{i}, \mu\right)\right), z\left(\tau_{i}-0\right), \mu\right)
\end{gathered}
$$

and

$$
\begin{gathered}
z(t)=V\left(t, t_{0}, \mu\right) z+\int_{t_{0}}^{t} V(t, \tau, \mu) g\left(x_{0}(\tau)\right. \\
\left.+v_{1}\left(\tau, x_{0}(\tau), z(\tau), \theta(\tau, \mu)\right), z(\tau), \theta(\tau, \mu)\right) d \tau \\
+\sum_{t_{0} \leq \tau_{i}<t} V\left(t, \tau_{i}, \mu\right) K_{i}\left(x_{0}\left(\tau_{i}-0\right)\right. \\
\left.+v_{1}\left(\tau_{i}-0, x_{0}\left(\tau_{i}-0\right), z\left(\tau_{i}-0\right), \theta\left(\tau_{i}, \mu\right)\right), z\left(\tau_{i}-0\right), \mu\right)
\end{gathered}
$$

where $x_{0}(\tau)=U\left(\tau, t_{0}, \mu\right) x_{0}$.

To solve the functional equation (3.2) we introduce the operator $\mathbf{E}$ from $\mathbf{G}_{1}(p)$ to $\mathbf{B}_{1}$ by the formula:

$$
\begin{gathered}
\mathbf{E} v_{1}\left(t_{0}, x_{0}, z, \theta\left(t_{0}, \mu\right)\right)=-\int_{t_{0}}^{+\infty} U\left(t_{0}, \tau, \mu\right) f\left(x_{0}(\tau)\right. \\
\left.+v_{1}\left(\tau, x_{0}(\tau), z(\tau), \theta(\tau, \mu)\right), z(\tau), \theta(\tau, \mu)\right) d \tau \\
-\sum_{t_{0} \leq \tau_{i}} U\left(t_{0}, \tau_{i}, \mu\right) I_{i}\left(x_{0}\left(\tau_{i}-0\right)\right. \\
\left.+v_{1}\left(\tau_{i}-0, x_{0}\left(\tau_{i}-0\right), z\left(\tau_{i}-0\right), \theta\left(\tau_{i}, \mu\right)\right), z\left(\tau_{i}-0\right), \mu\right)
\end{gathered}
$$

and

$$
z(t)=V\left(t, t_{0}, \mu\right) z+\int_{t_{0}}^{t} V(t, \tau, \mu) g\left(x_{0}(\tau)\right.
$$




$$
\begin{gathered}
\left.+v_{1}\left(\tau, x_{0}(\tau), z(\tau), \theta(\tau, \mu)\right), z(\tau), \theta(\tau, \mu)\right) d \tau \\
+\sum_{t_{0} \leq \tau_{i}<t} V\left(t, \tau_{i}, \mu\right) K_{i}\left(x_{0}\left(\tau_{i}-0\right)\right. \\
\left.+v_{1}\left(\tau_{i}-0, x_{0}\left(\tau_{i}-0\right), z\left(\tau_{i}-0\right), \theta\left(\tau_{i}, \mu\right)\right), z\left(\tau_{i}-0\right), \mu\right) .
\end{gathered}
$$

Let us estimate the difference $\left\|\mathbf{E} v_{1}\left(t_{0}, x_{0}, z, \mu\right)-\mathbf{E} v_{1}^{\prime}\left(t_{0}, x_{0}, z^{\prime}, \mu\right)\right\|$, taking into consideration the properties of $f, g, I_{i}, K_{i}, v_{1}$. After mathematical transformations we obtain

$$
\left\|\mathbf{E} v_{1}\left(t_{0}, x_{0}, z, \mu\right)-\mathbf{E} v_{1}^{\prime}\left(t_{0}, x_{0}, z^{\prime}, \mu\right)\right\| \leq p\left|z-z^{\prime}\right|+\varepsilon M(p+1)\left\|v_{1}-v_{1}^{\prime}\right\| .
$$

If $2 \varepsilon M<1+\sqrt{1-4 \varepsilon L}$, then $\varepsilon M(p+1)<1$. We conclude that $\mathbf{E}$ is a contraction. It involves there is only one solution in $\mathbf{G}_{1}(p)$, satisfying the functional equation (3.2).

Next, we construct the map:

$$
H_{1}\left(t_{0}, x_{0}, z, \mu\right)=\left(h_{1}\left(t_{0}, x_{0}, z, \mu\right), z\right)=\left(x_{0}+v_{1}\left(t_{0}, x_{0}, z, \theta\left(t_{0}, \mu\right)\right), z\right) .
$$

It can be easily checked that:

$$
H_{1}\left(t, \Psi\left(t, t_{0}, x_{0}, y_{0}, \mu\right), \theta\left(t-t_{0}, \mu\right)\right)=\Phi\left(t, t_{0}, H_{1}\left(t_{0}, x_{0}, y_{0}, \mu\right), \mu\right) .
$$

Step 2. In the same space $\mathbf{G}_{1}(p)$ we define the map by the following formula:

$$
\begin{gathered}
v_{2}\left(t_{0}, x_{0}, y_{0}, \theta\left(t_{0}, \mu\right)\right)=\int_{t_{0}}^{+\infty} U\left(t_{0}, \tau, \mu\right) f\left(\Phi\left(\tau, t_{0}, x_{0}, y_{0}, \mu\right), \theta(\tau, \mu)\right) d \tau \\
\quad+\sum_{t_{0} \leq \tau_{i}} U\left(t_{0}, \tau_{i}, \mu\right) I_{i}\left(\Phi\left(\tau_{i}-0, t_{0}, x_{0}, y_{0}, \mu\right), \mu\right)
\end{gathered}
$$

Next, we compute that

$$
\begin{gathered}
v_{2}\left(t, \Phi\left(t, t_{0}, x_{0}, y_{0}, \mu\right), \theta(t, \mu)\right) \\
=\int_{t}^{+\infty} U(t, \tau, \mu) f\left(\Phi\left(\tau, t_{0}, x_{0}, y_{0}, \mu\right), \theta(\tau, \mu)\right) d \tau \\
+\sum_{t \leq \tau_{i}} U\left(t, \tau_{i}, \mu\right) I_{i}\left(\Phi\left(\tau_{i}-0, t_{0}, x_{0}, y_{0}, \mu\right), \mu\right) .
\end{gathered}
$$

We construct the map:

$$
H_{2}\left(t_{0}, x_{0}, y_{0}, \mu\right)=\left(h_{2}\left(t_{0}, x_{0}, y_{0}, \mu\right), y_{0}\right)=\left(x_{0}+v_{2}\left(t_{0}, x_{0}, y_{0}, \theta\left(t_{0}, \mu\right)\right), y_{0}\right)
$$


Applying the (3.3), we conclude that

$$
h_{2}\left(t, \Phi\left(t, t_{0}, x_{0}, y_{0}, \mu\right), \theta\left(t-t_{0}, \mu\right)\right)=U\left(t, t_{0}, \mu\right) h_{2}\left(t_{0}, x_{0}, y_{0}, \mu\right) .
$$

Step 3. Let us prove that $H_{2}\left(t_{0}, H_{1}\left(t_{0}, x_{0}, y_{0}, \mu\right), \mu\right)=\left(x_{0}, y_{0}\right)$. It is sufficiently to check up that

$$
\begin{gathered}
h_{2}\left(t_{0}, H_{1}\left(t_{0}, x_{0}, y_{0}, \mu\right), \mu\right) \\
=h_{1}\left(t_{0}, x_{0}, y_{0}, \mu\right)+v_{2}\left(t_{0}, H_{1}\left(t_{0}, x_{0}, y_{0}, \mu\right), \theta\left(t_{0}, \mu\right)\right) \\
=x_{0}+v_{1}\left(t_{0}, x_{0}, y_{0}, \theta\left(t_{0}, \mu\right)\right)+v_{2}\left(t_{0}, H_{1}\left(t_{0}, x_{0}, y_{0}, \mu\right), \theta\left(t_{0}, \mu\right)\right) \\
=x_{0}-\int_{t_{0}}^{+\infty} U\left(t_{0}, \tau, \mu\right) f\left(\Phi\left(\tau, t_{0}, H_{1}\left(t_{0}, x_{0}, y_{0}, \mu\right), \mu\right), \theta(\tau, \mu)\right) d \tau \\
-\sum_{t_{0} \leq \tau_{i}} U\left(t_{0}, \tau_{i}, \mu\right) I_{i}\left(\Phi\left(\tau_{i}-0, t_{0}, H_{1}\left(t_{0}, x_{0}, y_{0}, \mu\right), \mu\right), \mu\right) \\
+\int_{t_{0}}^{+\infty} U\left(t_{0}, \tau, \mu\right) f\left(\Phi\left(\tau, t_{0}, H_{1}\left(t_{0}, x_{0}, y_{0}, \mu\right), \mu\right), \theta(\tau, \mu)\right) d \tau \\
+\sum_{t_{0} \leq \tau_{i}} U\left(t_{0}, \tau_{i}, \mu\right) I_{i}\left(\Phi\left(\tau_{i}-0, t_{0}, H_{1}\left(t_{0}, x_{0}, y_{0}, \mu\right), \mu\right), \mu\right)=x_{0} .
\end{gathered}
$$

Step 4. Now let us prove $H_{1}\left(t_{0}, H_{2}\left(t_{0}, x_{0}, y_{0}, \mu\right), \mu\right)=\left(x_{0}, y_{0}\right)$. Let us consider the Banach space $\mathbf{B}_{2}$ of the bounded maps that are continuous for $(t, x, y, z, \mu) \in\left(\tau_{i}, \tau_{i+1}\right] \times \mathbf{X} \times \mathbf{Y} \times \mathbf{Z} \times \mathbf{M}$ and have first kind breaks for $t=\tau_{i}$ :

$$
\mathbf{B}_{2}=\left\{v \mid v: \mathbb{R} \times \mathbf{X} \times \mathbf{Y} \times \mathbf{Z} \rightarrow \mathbf{X} \text { and } \sup _{t, x, y, z}|v(t, x, y, z, \mu)|<\infty\right\}
$$

with the norm $\|v\|=\sup _{t, x, y, z, \mu}|v(t, x, y, z, \mu)|$.

Let us introduce the set:

$$
\mathbf{G}_{2}(p)=\left\{v \in \mathbf{B}_{2}|| v(t, x, y, z, \mu)-v\left(t, x, y, z^{\prime}, \mu\right)|\leq p| z-z^{\prime} \mid\right\} .
$$

In the space $\mathbf{G}_{2}(p)$ we consider the functional equation:

$$
\begin{gathered}
v_{3}\left(t_{0}, x_{0}, y_{0}, z, \theta\left(t_{0}, \mu\right)\right)=\int_{t_{0}}^{+\infty} U\left(t_{0}, \tau, \mu\right)(f(\Phi(\tau), \theta(\tau, \mu)) \\
\left.-f\left(x(\tau)+v_{3}(\tau, \Phi(\tau), z(\tau), \theta(\tau, \mu)), z(\tau), \theta(\tau, \mu)\right)\right) d \tau
\end{gathered}
$$




$$
\begin{gathered}
+\sum_{t_{0} \leq \tau_{i}} U\left(t_{0}, \tau_{i}, \mu\right)\left(I_{i}\left(\Phi\left(\tau_{i}-0\right), \mu\right)-I_{i}\left(x\left(\tau_{i}-0\right)\right.\right. \\
\left.\left.+v_{3}\left(\tau_{i}-0, \Phi\left(\tau_{i}-0\right), z\left(\tau_{i}-0\right), \theta\left(\tau_{i}, \mu\right)\right), z\left(\tau_{i}-0\right), u\right)\right)
\end{gathered}
$$

and

$$
\begin{gathered}
z(t)=V\left(t, t_{0}, \mu\right) z+\int_{t_{0}}^{t} V(t, \tau, \mu) g(\tau, x(\tau) \\
\left.+v_{3}(\tau, \Phi(\tau), z(\tau), \theta(\tau, \mu)), z(\tau), \theta(\tau, \mu)\right) d \tau \\
+\sum_{t_{0} \leq \tau_{i}<t} V\left(t_{0}, \tau_{i}, \mu\right) K_{i}\left(x\left(\tau_{i}-0\right)\right. \\
\left.+v_{3}\left(\tau_{i}-0, \Phi\left(\tau_{i}-0\right), z\left(\tau_{i}-0\right), \theta\left(\tau_{i}, \mu\right)\right), z\left(\tau_{i}-0\right), \mu\right) .
\end{gathered}
$$

We introduce the operator $\mathbf{E}$ :

$$
\begin{gathered}
\mathbf{E} v_{3}\left(t_{0}, x_{0}, y_{0}, z, \theta\left(t_{0}, \mu\right)\right)=\int_{t_{0}}^{+\infty} U\left(t_{0}, \tau, \mu\right)(f(\Phi(\tau), \theta(\tau, \mu)) \\
\left.-f\left(x(\tau)+v_{3}(\tau, \Phi(\tau), z(\tau), \theta(\tau, \mu)), z(\tau), \theta(\tau, \mu)\right)\right) d \tau \\
\quad+\sum_{t_{0} \leq \tau_{i}} U\left(t_{0}, \tau_{i}, \mu\right)\left(I_{i}\left(\Phi\left(\tau_{i}-0\right), \mu\right)-I_{i}\left(x\left(\tau_{i}-0\right)\right.\right. \\
\left.\left.+v_{3}\left(\tau_{i}-0, \Phi\left(\tau_{i}-0\right), z\left(\tau_{i}-0\right), \theta\left(\tau_{i}, \mu\right)\right), z\left(\tau_{i}-0\right), \mu\right)\right) .
\end{gathered}
$$

In the same manner as we produced in the first step we determine the difference $\left\|\mathbf{E} v_{3}-\mathbf{E} v_{3}^{\prime}\right\|$. We take the same decisions and finally obtain, that $\mathbf{E}$ is a contraction in $\mathbf{G}_{2}(p)$. There is only one solution for the functional equation (3.5). Next, we construct the map:

$H_{3}\left(t_{0}, x_{0}, y_{0}, z, \mu\right)=\left(h_{3}\left(t_{0}, x_{0}, y_{0}, z, \mu\right), z\right)=\left(x_{0}+v_{3}\left(t_{0}, x_{0}, y_{0}, z, \theta\left(t_{0}, \mu\right)\right), z\right)$.

We notice that the map

$$
\begin{gathered}
\alpha\left(t_{0}, x_{0}, y_{0}, z, \theta\left(t_{0}, \mu\right)\right) \\
=v_{2}\left(t_{0}, x_{0}, y_{0}, \theta\left(t_{0}, \mu\right)\right)+v_{1}\left(t_{0}, h_{2}\left(t_{0}, x_{0}, y_{0}, \mu\right), z, \theta\left(t_{0}, \mu\right)\right)
\end{gathered}
$$

also satisfies the functional equation (3.5) and $\alpha \in \mathbf{G}_{2}(p)$, therefore

$$
\begin{gathered}
v_{3}\left(t_{0}, x_{0}, y_{0}, z, \theta\left(t_{0}, \mu\right)\right) \\
=v_{2}\left(t_{0}, x_{0}, y_{0}, \theta\left(t_{0}, \mu\right)\right)+v_{1}\left(t_{0}, h_{2}\left(t_{0}, x_{0}, y_{0}, \mu\right), z, \theta\left(t_{0}, \mu\right)\right) .
\end{gathered}
$$


Now we make equal the third and the fourth argument of $v_{3}$ and put them into the expression.

We obtain that $v_{3}\left(t_{0}, x_{0}, y_{0}, y_{0}, \theta\left(t_{0}, \mu\right)\right)=0$.

Therefore $H_{1}\left(t_{0}, H_{2}\left(t_{0}, x_{0}, y_{0}, \mu\right), \mu\right)=\left(x_{0}, y_{0}\right)$.

$$
\begin{gathered}
h_{1}\left(t_{0}, H_{2}\left(t_{0}, x_{0}, y_{0}, \mu\right), \mu\right)=h_{2}\left(t_{0}, x_{0}, y_{0}, \mu\right)+v_{1}\left(t_{0}, H_{2}\left(t_{0}, x_{0}, y_{0}, \mu\right), \theta\left(t_{0}, \mu\right)\right) \\
=x_{0}+v_{2}\left(t_{0}, x_{0}, y_{0}, \theta\left(t_{0}, \mu\right)\right)+v_{1}\left(t_{0}, H_{2}\left(t_{0}, x_{0}, y_{0}, \mu\right), \theta\left(t_{0}, \mu\right)\right)=x_{0} .
\end{gathered}
$$

We get that $H_{1}(t, \cdot, \cdot, \mu)$ is a homeomorphism establishing dynamical equivalence of systems (2.1) and (2.4).

\section{REFERENCES}

[1] D. D. Bainov and S. I. Kostadinov, Abstract Impulsive Differential Equations, Descartes Press, Koriyama, 1996.

[2] A. Reinfelds, Dynamical equivalence of impulsive differential equations, Nonlinear Anal. 30 (1997), No. 5, 2743-2752.

[3] A. Reinfelds, A reduction theorem for systems of differential equations with impuse effect in a Banach space, J. Math. Anal. Appl. 203 (1996), No. 1, 187-210.

[4] A. M. Samoilenko and N. A. Perestyuk, Impulsive Differential Equations, World Sci. Publishing, River Edge, NJ, 1995.

[5] L. Sermone, Equivalence of semilinear dynamical extentions with impulse effect, in S. Bilchev and S. Terzian (eds.), Differential equations and applications. Proceedings of the fifth international conference on differential equations and applications, Rousse, Bulgaria, August 24-29, 1995. Union of Bulgarian Mathematicians, Rousse, 1995, 103110.

[6] L. Sermone, Reduction of differential equations with impulse effect. J. Appl. Math. Stochastic Anal. 10 (1997), No. 1, 79-87. 